\title{
Tumour necrosis factor-a/interleukin-10 ratio in patients with obstructive sleep apnoea hypopnoea syndrome
}

\author{
$\mathrm{H} \mathrm{JIANG}^{1}, \mathrm{H} \mathrm{CAO}^{1}, \mathrm{P}_{\mathrm{WANG}}{ }^{2}, \mathrm{~W} \mathrm{LIU}^{3}, \mathrm{~F} \mathrm{CAO}^{2}, \mathrm{~J} \mathrm{CHEN}^{2}$ \\ ${ }^{1}$ Clinical Laboratory, ${ }^{2}$ Department of Otolaryngology, and ${ }^{3}$ Department of Cardiology, Xiangyang Central \\ Hospital Affiliated to Hubei University of Arts and Science, Xiangyang, PR China
}

\begin{abstract}
Objective: To explore the significance of the tumour necrosis factor- $\alpha /$ interleukin-10 ratio and the effect of continuous positive airway pressure in patients with different degrees of obstructive sleep apnoea hypopnoea syndrome severity.

Method: This study comprised 135 patients with obstructive sleep apnoea hypopnoea syndrome and 94 control subjects.

Results: Tumour necrosis factor- $\alpha$ and tumour necrosis factor- $\alpha$ /interleukin-10 ratio values were significantly higher in the obstructive sleep apnoea hypopnoea syndrome group than in the control group, but interleukin-10 was significantly lower. Tumour necrosis factor- $\alpha$ /interleukin-10 ratio values increased in line with the severity of obstructive sleep apnoea hypopnoea syndrome. In multivariate analysis, the tumour necrosis factor- $\alpha /$ interleukin-10 ratio correlated positively with the apnoea-hypopnoea index and all indices of obstructive sleep apnoea hypopnoea syndrome, except for age, body mass index and neck circumference. After one month of continuous positive airway pressure therapy, levels of tumour necrosis factor- $\alpha$ decreased; interleukin-10 showed no change.

Conclusion: The results suggest that inflammation is activated and anti-inflammatory cytokines are decreased in obstructive sleep apnoea hypopnoea syndrome patients. Tumour necrosis factor- $\alpha$ /interleukin-10 ratio may prove useful for severity monitoring and management of obstructive sleep apnoea hypopnoea syndrome patients, and may reduce the need for polysomnography.
\end{abstract}

Key words: Sleep Apnea, Obstructive; Polysomnography; Tumor Necrosis Factor-Alpha; Interleukin-10

\section{Introduction}

Obstructive sleep apnoea hypopnoea syndrome (OSAHS) is characterised by recurrent episodes of complete or partial obstruction of the upper airway during sleep. This leads to oxygen desaturation, fragmented sleep and daytime somnolence. ${ }^{1}$ For people with sleep apnoea, the combination of disturbed sleep and oxygen starvation may lead to hypertension, heart disease, and mood and memory problems. ${ }^{2}$ Sleep apnoea also increases the risk of automobile crashes. ${ }^{3}$ It is therefore important to assess the risk of OSAHS early.

There is evidence that OSAHS is associated with a group of proinflammatory cytokines. Prior studies have shown that biomarkers of inflammation are raised in apnoeic patients. ${ }^{4}$ These inflammatory changes are postulated to occur, in part, due to snoring that evokes vibration frequencies associated with soft-tissue damage (local inflammation). ${ }^{4}$ In addition, there is evidence of systemic inflammation in patients with OSAHS.

Tumour necrosis factor- $\alpha$ (TNF- $\alpha$ ) is a proinflammatory cytokine chiefly produced by activated macrophages, although it can be produced by many other cell types, such as cluster of differentiation $4+$ lymphocytes, natural killer cells and neurons. ${ }^{5}$ It is associated with a variety of immunological functions such as the regulation of cellular immune responses and modulation of the expression of many other cytokines. ${ }^{5}$

The production of TNF- $\alpha$, which can be attributed to obstructive sleep apnoea (OSA)-induced hypoxic stress, is mediated by nuclear factor $\kappa \beta$ activation. ${ }^{6}$ The prevailing T-helper type 1 (Th1) cytokine pattern of the immune cells in the peripheral blood of OSAHS patients can partially be explained by hypoxia. In fact, experimental evidence supports the role of hypoxia in inducing the expression of the 
proinflammatory cytokines, in particular TNF- $\alpha$, but also interleukin (IL)-1b and IL-6. ${ }^{7}$

Interleukin-10 is one of the most important antiinflammatory cytokines. It is produced mainly by T-helper type 2 (Th2) lymphocytes, macrophages, B cells and monocytes. Convincing evidence suggests that IL-10 is crucial for atherosclerotic plaque stability, whereby IL-10 blocks the synthesis of proinflammatory cytokines. ${ }^{8}$

It has been revealed that positive airway pressure treatment significantly lessens the symptoms of OSAHS and related diseases; it is a gold standard therapy for moderate and severe OSAHS.'

The present study aimed to explore the significance of the TNF- $\alpha /$ IL-10 ratio and the effect of continuous positive airway pressure (CPAP) in patients with different degrees of OSAHS severity.

\section{Materials and methods}

\section{Study population}

The study included 135 adult OSAHS patients, all of whom had visited the chest or otolaryngology clinics at the Xiangyang Central Hospital Affiliated to Hubei University of Arts and Science, from January 2008 to January 2012, for the evaluation of sleep problems. All patients underwent diagnostic polysomnography in the sleep laboratory to confirm OSAHS and determine the severity of the condition. We also enrolled 94 control volunteers, matched in terms of age and body mass index (BMI), who were undergoing routine physical examination in our hospital during the same period as the patients.

Participants were excluded from the present study if they had: chronic obstructive pulmonary disease, active inflammatory disease or autoimmune disorders, severe heart failure, advanced renal or hepatic disease, personal or family history of psychiatric disorders, or previously documented coronary artery disease. All patients gave written informed consent before serum sample collection. The study protocol was approved by the ethics committee of our hospital.

\section{Polysomnography}

All subjects underwent overnight polysomnography using the Sandman Elite ${ }^{\circledR}$ sleep diagnostic system. The subjects were advised not to consume caffeinecontaining beverages or take medication that might affect their sleeping pattern. Polysomnographic monitoring was performed using a standard technique.

Apnoeas were defined as the complete cessation of airflow for 10 seconds or longer. Hypopnoea was defined as a reduction in airflow with a 50 per cent fall from baseline for at least 10 seconds, accompanied by a 3 per cent drop in oxygen saturation from the preceding stable saturation level or event-associated arousal. The apnoea-hypopnoea index (AHI) reflected the number of apnoeas and hypopnoeas per hour of sleep. The diagnosis of OSAHS was based on an
AHI of more than 5. Patients were subdivided into mild (AHI $=5-14)$, moderate $(\mathrm{AHI}=15-29)$ and severe $(\mathrm{AHI}=30$ or higher $)$ OSAHS groups.

\section{Tumour necrosis factor- $\alpha$ and interleukin-10 measurement}

All subjects went to bed at 21.00 and were awakened at 06.00. Serum samples were obtained at 07.00. Samples were collected after the initial polysomnography, and after one month of CPAP therapy in OSAHS patients who underwent this treatment.

Following centrifugation, the sera were stored at $-80^{\circ} \mathrm{C}$ until analysed. Tumour necrosis factor- $\alpha$ (Biovision, Milpitas, California, USA) and interleukin (IL)-10 (Biovision) were measured by enzyme-linked immunosorbent assay methods.

\section{Continuous positive airway pressure treatment}

Ninety suitable patients with moderate to severe OSAHS, who had agreed to possible CPAP therapy before undergoing diagnostic sleep study, began nasal CPAP therapy within one month after polysomnography. All were evaluated for symptoms and side effects, and objective compliance data were downloaded from the CPAP devices.

\section{Statistical analysis}

Data normality was analysed using the Kolmogorov-Smirnov test. Differences between the two groups were analysed using the unpaired $t$ test, Mann-Whitney $U$ test or chi-square test, as indicated. Differences among the groups were analysed by one-way analysis of variance followed by the Tukey post-hoc test or the Kruskal-Wallis analysis when appropriate. Pearson's correlation analysis was used to assess correlations between the tumour necrosis factor- $\alpha /$ IL-10 ratio and baseline and polysomnography variables. Analyses were performed using the Statistical Package for the Social Sciences $\left(\right.$ SPSS $\left.^{\circledR}\right)$ software (version 13.0). A value of $p<0.05$ (two-tailed) was considered statistically significant.

Continuous, normally distributed variables are presented as means \pm standard deviations. Categorical data are presented as numbers and percentages.

\section{Results}

\section{Baseline characteristics}

A total of 135 OSAHS patients and 94 control subjects were enrolled in the present study. The baseline characteristics and clinical profiles of both groups are shown in Table I.

There were no differences in age, sex or BMI between the OSAHS patients and controls. The mean age was $48.7 \pm 12.1$ years in the OSAHS group and $47.2 \pm 13.5$ years in the control group. Of the 135 OSAHS patients, 80 were male and 55 were female. In the control group, 55 of the 94 subjects were male 


\begin{tabular}{|c|c|c|c|}
\hline \multicolumn{4}{|c|}{$\begin{array}{l}\text { TABLE I } \\
\text { 3ASELINE CHARACTERISTICS AND CLINICAL PROFILES }\end{array}$} \\
\hline Variable & OSAHS group* & Control group ${ }^{\dagger}$ & $p$ \\
\hline Sex (M:F ratio) & $80: 55$ & $55: 39$ & 0.62 \\
\hline Age (years) & $48.7 \pm 12.1$ & $47.2 \pm 13.5$ & 0.75 \\
\hline $\mathrm{BMI}\left(\mathrm{kg} / \mathrm{m}^{2}\right)$ & $27.48 \pm 2.56$ & $27.52 \pm 2.58$ & 0.83 \\
\hline Neck circumference $(\mathrm{cm})$ & $40.7 \pm 3.22$ & $36.8 \pm 2.76$ & 0.02 \\
\hline AHI (events/hour) & $24.57 \pm 15.9$ & $1.60 \pm 1.61$ & $<0.001$ \\
\hline Desaturation index & $23.7 \pm 22.4$ & $5.7 \pm 5.2$ & $<0.001$ \\
\hline Lowest $\mathrm{O}_{2}$ sat $(\%)$ & $73.2 \pm 12.5$ & $88.5 \pm 6.8$ & $<0.001$ \\
\hline Mean $\mathrm{O}_{2}$ sat $(\%)$ & $87.4 \pm 3.8$ & $91.4 \pm 1.5$ & $<0.001$ \\
\hline$\%$ Total time slept in $<90 \% \mathrm{O}_{2}$ sat & $15 \pm 7$ & $0.14 \pm 0.7$ & $<0.001$ \\
\hline TNF- $\alpha(n g / 1)$ & $765.77 \pm 64.04$ & $232.24 \pm 31.5$ & $<0.001$ \\
\hline IL-10 (ng/1) & $5.68 \pm 4.02$ & $8.76 \pm 5.25$ & $<0.001$ \\
\hline TNF- $\alpha /$ IL-10 ratio & $134.82 \pm 15.93$ & $26.51 \pm 6.01$ & $<0.001$ \\
\hline
\end{tabular}

and 39 were female. The mean BMI values for the control group and OSAHS group were $27.52 \pm 2.58$ and $27.48 \pm 2.56$, respectively $(p=0.83)$.

The mean AHI was $24.57 \pm 15.9$ events per hour in OSAHS patients versus $1.60 \pm 1.61$ events per hour in the control group $(p<0.001)$. There were also significant differences between the groups in terms of desaturation index, lowest oxygen saturation percentage, mean oxygen saturation percentage and percentage of the total time slept in which oxygen saturation levels were lower than 90 per cent (all $p<0.001)$.

The plasma level of tumour necrosis factor- $\alpha$ (TNF- $\alpha$ ) was significantly higher in the OSAHS group than in the control group $(765.77 \pm 64.04 \mathrm{ng} / 1$ vs $232.24 \pm 31.5 \mathrm{ng} / 1 ; p<0.001)$. However, the interleukin (IL)-10 level was significantly lower in the OSAHS group than in the control group (5.68 \pm $4.02 \mathrm{ng} / 1$ vs $8.76 \pm 5.25 \mathrm{ng} / 1 ; p<0.001)$. The TNFa/IL-10 ratio was significantly higher in the OSAHS group than in the control group $(134.82 \pm 15.93 \mathrm{vs}$ $26.51 \pm 6.01 ; p<0.001)$.

\section{Biochemical characteristics according to severity}

As shown in Table II, the TNF- $\alpha$ level was significantly higher in the severe OSAHS group (836.72 \pm $71.06 \mathrm{ng} / 1)$ than in the moderate OSAHS group $(764.48 \pm 63.28 \mathrm{ng} / 1)$ or mild OSAHS group
$(545.36 \pm 54.06 \mathrm{ng} / 1)$. Thus, the TNF- $\alpha$ level was elevated with increased OSAHS severity. In contrast, the IL-10 level was reduced with increased OSAHS severity (mild group, $6.68 \pm 4.74 \mathrm{ng} / 1$; moderate group, $5.77 \pm 4.06 \mathrm{ng} / 1 ;$ severe group, $4.87 \pm 3.84 \mathrm{ng} / 1)$. When the three groups were compared, the severe OSAHS group had the highest TNF- $\alpha / \mathrm{IL}-10$ ratio value (severe group, $171.81 \pm 18.51$; moderate group, $132.49 \pm 15.59 ;$ mild group, $81.64 \pm 11.41 ; \quad p<$ $0.001)$.

\section{Correlation data}

A correlation was performed to investigate the relationship between the TNF- $\alpha /$ IL-10 ratio and the severity of OSAHS. As shown in Figure 1, a significant positive correlation was found between increments in TNF- $\alpha$ / IL-10 ratio and severity of OSAHS (ratio value was $81.64 \pm 11.41$ in the mild OSAHS group, $132.49 \pm$ 15.59 in the moderate OSAHS group and $171.81 \pm$ 18.51 in the severe OSAHS group; $p<0.001$ ).

Following log transformations of the TNF- $\alpha / \mathrm{IL}-10$ ratio values, we performed multiple comparisons among the three subgroups. The results showed that the TNF- $\alpha /$ IL-10 ratio values were significantly higher in the moderate OSAHS group $(p<0.01)$ and severe OSAHS group $(p<0.001)$ when compared with those of the mild OSAHS group.

\begin{tabular}{|c|c|c|c|c|}
\hline \multicolumn{5}{|c|}{$\begin{array}{c}\text { TABLE II } \\
\text { BIOCHEMICAL CHARACTERISTICS BY OSAHS SEVERITY }\end{array}$} \\
\hline Variable & Mild OSAHS* & Moderate OSAHS ${ }^{\dagger}$ & Severe OSAHS & $p$ \\
\hline AHI (events/hour) & $11.52 \pm 3.9$ & $21.06 \pm 8.64$ & $35.1 \pm 24.7$ & $<0.001$ \\
\hline TNF- $\alpha(n g / 1)$ & $545.36 \pm 54.06$ & $764.48 \pm 63.28$ & $836.72 \pm 71.06$ & $<0.001$ \\
\hline IL-10 (ng/1) & $6.68 \pm 4.74$ & $5.77 \pm 4.06$ & $4.87 \pm 3.84$ & $<0.001$ \\
\hline TNF- $\alpha /$ IL-10 ratio & $81.64 \pm 11.41$ & $132.49 \pm 15.59$ & $171.81 \pm 18.51$ & $<0.001$ \\
\hline
\end{tabular}

Values represent means \pm standard deviations, unless indicated otherwise. Significance was set at $p<0.05 .{ }^{*} n=45 ;{ }^{\dagger} n=44 ;{ }^{\ddagger} n=46$. OSAHS = obstructive sleep apnoea hypopnoea syndrome; AHI = apnoea-hypopnoea index; TNF- $\alpha=$ tumour necrosis factor- $\alpha$; IL-10 = interleukin 10 


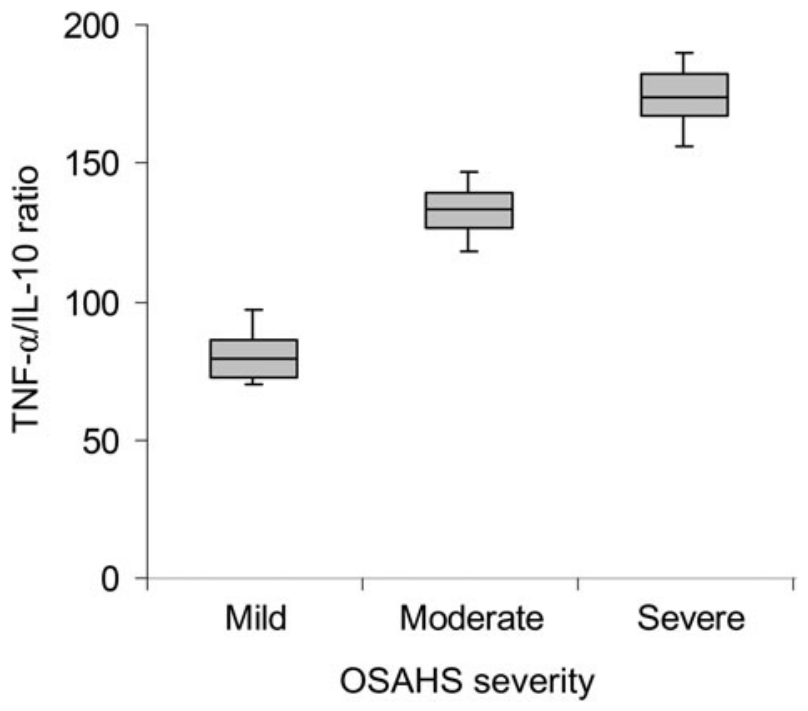

FIG. 1

Box-and-whisker plot showing tumour necrosis factor- $\alpha$ (TNF- $\alpha$ )/ interleukin-10 (IL-10) ratio in mild, moderate and severe obstructive sleep apnoea hypopnoea syndrome (OSAHS) patients. Boxes and error bars represent means and standard deviations respectively.

The associations between the log TNF- $\alpha /$ IL-10 ratio and other variables are summarised in Table III. Pearson's correlation analysis showed that the TNFa/IL-10 ratio correlated positively with AHI and all indices of OSAHS except for age, BMI and neck circumference (all $p<0.001$ ).

\section{Effect of continuous positive airway pressure therapy}

After the 90 CPAP-treated patients with severe OSAHS had received 1 month of CPAP therapy, AHI fell from $35.1 \pm 24.7$ to $9.1 \pm 4.2(p<0.001$ compared with pre-CPAP levels; Figure 2). The CPAP therapy led to a significant decrease in TNF- $\alpha$ levels (from $836.72 \pm 71.06$ to $418.2 \pm 42.1 \mathrm{ng} / 1 ; \quad p<0.001)$ (Figure 3 ), but this same course of CPAP therapy had

\begin{tabular}{lcc}
\multicolumn{3}{c}{ TABLE III } \\
\multicolumn{3}{c}{ CORRELATIONS BETWEEN LOG TNF- $\alpha$ /IL-10 } \\
AND CLINICAL CHARACTERISTICS* \\
\hline Variable & Log TNF- $\alpha /$ IL-10 ratio \\
\cline { 2 - 3 } & $R$ & $p$ \\
\hline Age & 0.05 & 0.65 \\
BMI & 0.06 & 0.50 \\
Neck circumference & 0.08 & 0.42 \\
Log AHI & 0.39 & $<0.001$ \\
Log desaturation index & 0.52 & $<0.001$ \\
Log lowest $\mathrm{O}_{2}$ sat & -0.36 & $<0.001$ \\
Log mean $\mathrm{O}_{2}$ sat & -0.38 & $<0.001$ \\
\% Total time slept in $<90 \% \mathrm{O}_{2}$ sat & 0.63 & $<0.001$ \\
Log TNF- $\alpha$ & 0.47 & $<0.001$ \\
Log IL-10 & -0.51 & $<0.001$ \\
\hline
\end{tabular}

Significance was set at $p<0.05 .{ }^{*}$ Using Spearman's rank correlation coefficients. TNF- $\alpha=$ tumour necrosis factor- $\alpha$; IL-10 $=$ interleukin 10; $\mathrm{BMI}=$ body mass index; $\mathrm{AHI}=$ apnoea-hypopnoea index; $\mathrm{O}_{2}$ sat $=$ oxygen saturation no significant effect on IL-10 levels $(p>0.05)$ (Figure 4).

\section{Discussion}

Obstructive sleep apnoea hypopnoea syndrome (OSAHS) should be considered a systemic disease rather than a local abnormality. Although the exact mechanism of OSAHS is not yet fully elucidated, inflammation is now thought to play a crucial role in the pathogenesis of the disease, and there is evidence that patients with OSAHS have increased interleukin (IL)-6, tumour necrosis factor- $\alpha$ (TNF- $\alpha$ ) and C-reactive protein levels. ${ }^{10-12}$

One study concluded that the neutralising of TNF- $\alpha$ with etanercept, which is a TNF- $\alpha$ antagonist, is associated with a significant reduction in objective sleepiness in obese patients with OSA. ${ }^{13}$ Another report showed that circulating levels of IL-6 and TNF- $\alpha$ were significantly elevated in OSA patients, and the levels of these cytokines were related to the severity of OSA. ${ }^{14}$ In the present study, we found that the TNF- $\alpha$ /IL-10 ratio and TNF- $\alpha$ levels were significantly higher in OSAHS patients compared with controls. We also investigated the correlation between increments in TNF- $\alpha /$ IL-10 ratio and OSAHS severity. Among the three subgroups of OSAHS patients, TNF- $\alpha /$ IL-10 ratio and TNF-a levels increased with the severity of the disease.

The relationship between the TNF- $\alpha$ /IL-10 ratio and OSAHS suggests a crucial role of activated inflammation in the pathogenesis of OSAHS. Therefore, inflammatory cytokines represent the potential to be assessed as biomarkers for OSAHS, owing to the inflammatory nature of the disease. These disease biomarkers could possibly provide information related to diagnosis, severity, prognosis and response to treatment. In fact, there has been a plethora of work conducted on inflammatory markers in subjects with OSA.

Tumour necrosis factor- $\alpha$, a Th1 cytokine, acted as a proinflammatory cytokine, whereas IL-10, one of the most important Th2 cytokines, acted as an anti-inflammatory cytokine. In the OSAHS group, TNF- $\alpha$ was significantly higher but IL-10 was lower than in the control group. Our findings suggest a superior Th1biased inflammatory response but suppressive $\mathrm{Th} 2$ anti-inflammatory response in OSAHS patients.

The mechanisms by which inflammation contributes to OSAHS are not known. Re-oxygenation after a brief period of hypoxia, as experienced repetitively and systematically by OSAHS patients, may predispose these individuals to cell stress. It has been suggested that such events favour the activation of a proinflammatory response as mediated through the nuclear transcription factor nuclear factor $\kappa \beta$, a master regulator of inflammatory gene expression. The findings of the current study suggest either that: a more substantial or a different pattern of hypoxaemia might be necessary to activate systemic inflammation; the system may need to be primed before hypoxic exposure; or increases in 


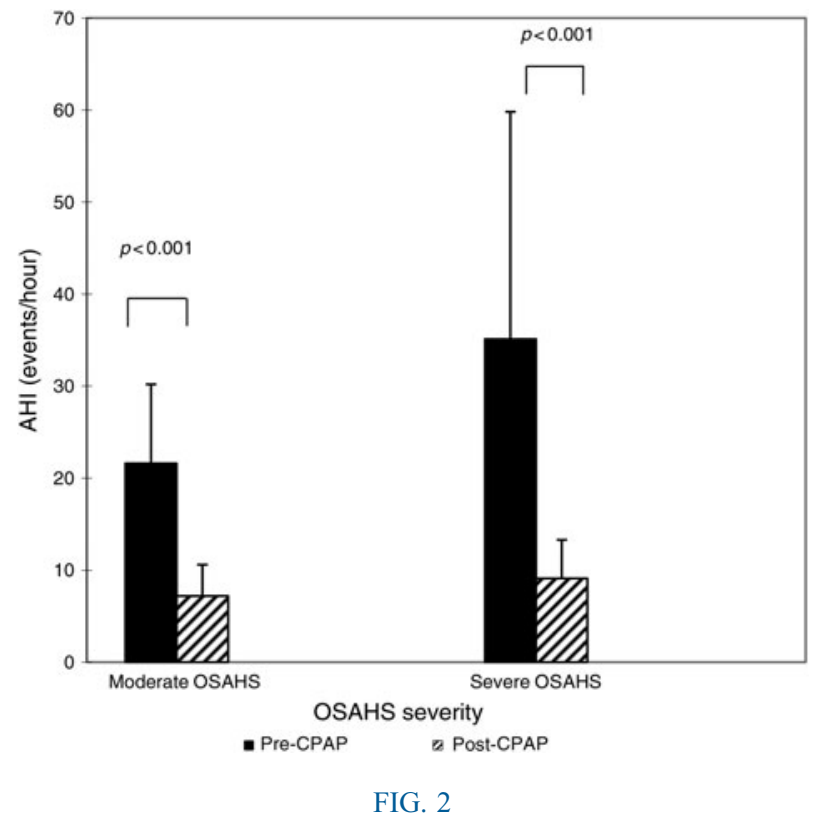

Impact of one month of continuous positive airway pressure (CPAP) therapy on apnoea-hypopnoea index (AHI) in obstructive sleep apnoea hypopnoea syndrome (OSAHS) patients. Boxes and error bars represent means and standard deviations respectively.

inflammatory markers in OSAHS patients may be more related to other factors such as obesity or nocturnal arousal. $^{15}$

Studies from various laboratories and clinical environments suggest that OSAHS is an inflammatory disorder. The increase in proinflammatory cytokine production in OSAHS patients can have important consequences for patient outcomes, especially with regard to the increased risk for developing atherosclerosis, and cardiovascular and cerebrovascular diseases. ${ }^{16}$ In a

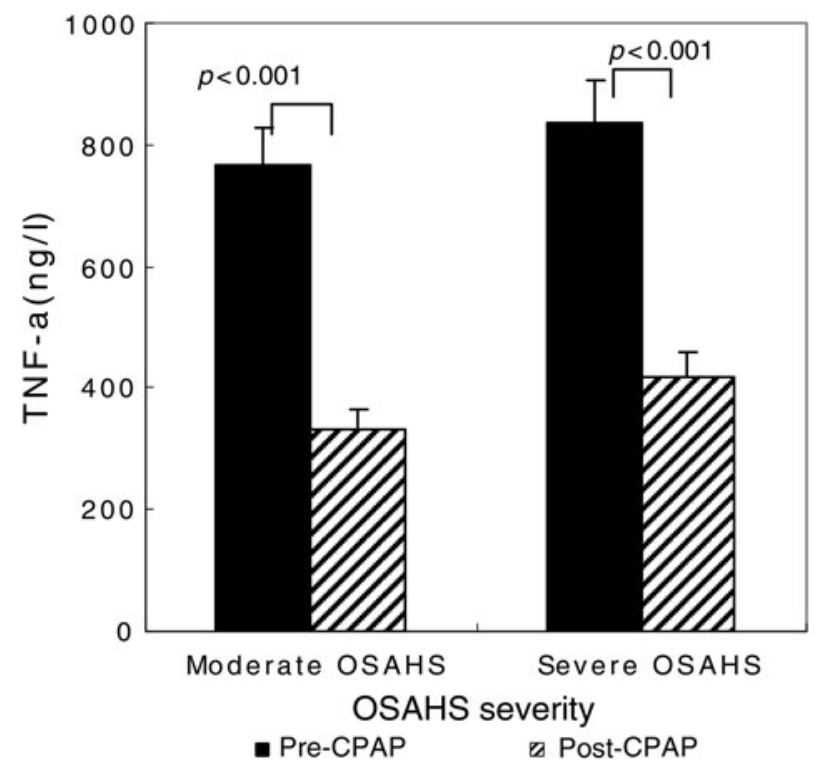

FIG. 3

Impact of one month of continuous positive airway pressure (CPAP) therapy on tumour necrosis factor- $\alpha$ (TNF- $\alpha$ ) levels in obstructive sleep apnoea hypopnoea syndrome (OSAHS) patients. Boxes and error bars represent means and standard deviations respectively.

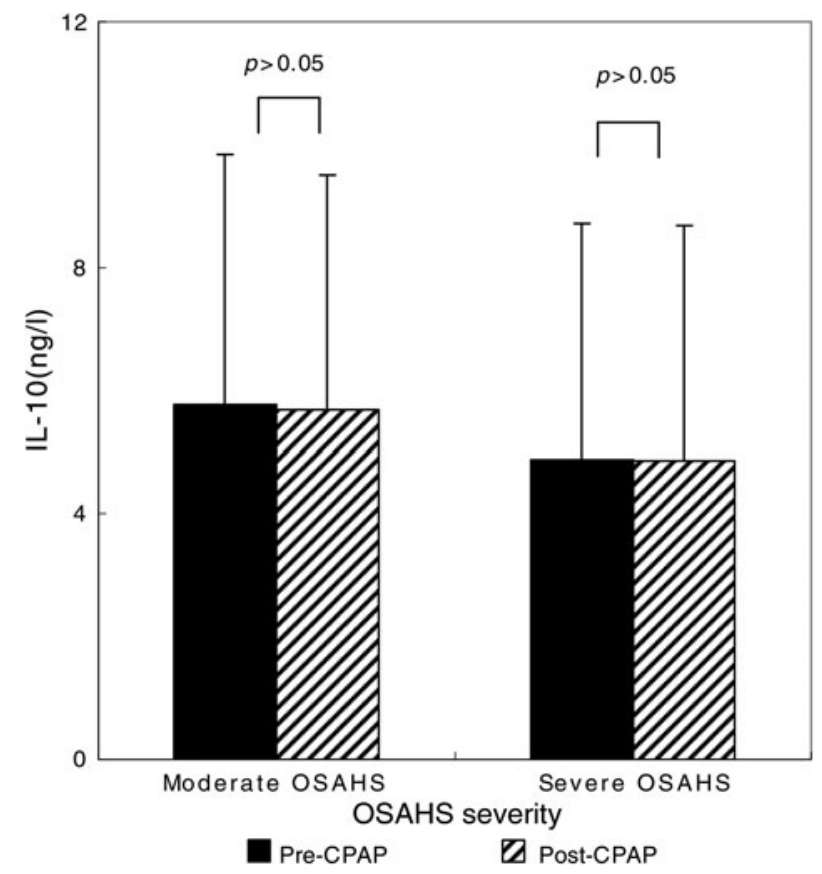

FIG. 4

Impact of one month of continuous positive airway pressure (CPAP) therapy on interleukin-10 (IL-10) levels in obstructive sleep apnoea hypopnoea syndrome (OSAHS) patients. Boxes and error bars represent means and standard deviations respectively.

meta-analysis performed to investigate the effect of CPAP therapy on systemic inflammation in patients with OSA, it was found that CPAP therapy could partially suppress systemic inflammation in OSA patients; furthermore, substantial differences were present among the various inflammatory markers. ${ }^{17}$

- Local and systemic inflammation is present in obstructive sleep apnoea hypopnoea syndrome (OSAHS)

- Inflammation was activated and antiinflammatory cytokines were decreased in OSAHS patients

- Continuous positive airway pressure therapy is an effective treatment to lower inflammatory response

Polysomnography is considered the gold standard diagnostic test for OSA. ${ }^{18}$ In-laboratory polysomnography is unsuitable for screening because of complexity, expense and inaccessibility. ${ }^{19}$ In our study, the severity of OSAHS was assessed using diagnostic polysomnography in a sleep laboratory. A Pearson's correlation analysis was then conducted to assess the correlations between the TNF- $\alpha$ /L-10 ratio and baseline and polysomnography variables. Clinical data can also be useful in identifying OSAHS. Furthermore, a previous study showed that a CPAP trial could predict sleep apnoea with a sensitivity of 80 per cent, a specificity of 97 per cent, and positive and negative predictive 
values of 97 per cent and 78 per cent, respectively. ${ }^{20}$ The findings suggested that polysomnography could have been avoided in 35 (46 per cent) of the 76 sleep apnoea patients with positive CPAP trial results. In our study, one month of CPAP therapy in the patients with severe OSAHS resulted in decreased AHI, with a corresponding significant decrease in TNF- $\alpha$ levels; however, this same course of CPAP therapy had no significant effect on IL-10 levels. It is concluded that one month of CPAP therapy had an appreciable effect on TNF- $\alpha$ and AHI parameters, but not on IL-10 levels.

The present study has some limitations. First, the number of participants was small; further studies with a larger population will elucidate the relationship between the TNF- $\alpha /$ IL-10 ratio and OSAHS. Second, only serum TNF- $\alpha$ and IL-10 levels were detected in the present study. Additional studies investigating other cytokines, such as Th1 and Th2 cytokines, might provide additional information on the disease.

\section{Acknowledgements}

This study was supported by grants from: the Hubei Natural Science Foundation of China (grant number 2012FFA071), the Xiangyang Science Foundation (grant number 2012[40]10) and the Sun Research Foundation (grant number 201202).

\section{References}

1 Feng X, Li P, Zhou C, Jia X, Kang J. Elevated levels of serum chemerin in patients with obstructive sleep apnea syndrome. Biomarkers 2012;17:248-53

2 Chandola T, Ferrie JE, Perski A, Akbaraly T, Marmot MG. The effect of short sleep duration on coronary heart disease risk is greatest among those with sleep disturbance: a prospective study from the Whitehall II cohort. Sleep 2010;33:739-44

3 George CF. Reduction in motor vehicle collisions following treatment of sleep apnoea with nasal CPAP. Thorax 2001;56: 508-12

4 Yokoe T, Minoguchi K, Matsuo H, Oda N, Minoguchi H, Yoshino $\mathrm{G}$ et al. Elevated levels of $\mathrm{C}$-reactive protein and interleukin-6 in patients with obstructive sleep apnea syndrome are decreased by nasal continuous positive airway pressure. Circulation 2003;107:1129-34

5 MacKenzie S, Planas JV, Goetz FW. LPS-stimulated expression of a tumor necrosis factor-alpha mRNA in primary trout monocytes and in vitro differentiated macrophages. Dev Comp Immunol 2003;27:393-400

6 Tamaki S, Yamauchi M, Fukuoka A, Makinodan K, Koyama N, Tomoda $\mathrm{K}$ et al. Production of inflammatory mediators by monocytes in patients with obstructive sleep apnea syndrome. Intern Med 2009;48:1255-62

7 Naldini A, Carraro F, Silvestri S, Bocci V. Hypoxia affects cytokine production and proliferative responses by human peripheral mononuclear cells. J Cell Physiol 1997;173:335-42
8 Grütz G. New insights into the molecular mechanism of interleukin-10-mediated immunosuppression. J Leukoc Biol 2005; 77:3-15

9 Koseoglu S, Ikinciogullari A, Cetin MA, Uysal GS, Kum RO, Arli B. The clinical efficiency of positive airway pressure treatment. ScientificWorldJournal 2013;2013:245476

10 Steiropoulos P, Papanas N, Nena E, Antoniadou M, Serasli E, Papoti S et al. Inflammatory markers in middle-aged obese subjects: does obstructive sleep apnea syndrome play a role? Mediators Inflamm 2010;2010:675320

11 Vgontzas AN, Papanicolaou DA, Bixler EO, Lotsikas A, Zachman K, Kales A et al. Circadian interleukin-6 secretion and quantity and depth of sleep. J Clin Endocrinol Metab 1999;84:2603-7

12 Guven SF, Turkkani MH, Ciftci B, Ciftci TU, Erdogan Y. The relationship between high-sensitivity $\mathrm{C}$-reactive protein levels and the severity of obstructive sleep apnea. Sleep Breath 2012;16:217-21

13 Vgontzas AN, Zoumakis E, Lin HM, Bixler EO, Trakada G, Chrousos GP. Marked decrease in sleepiness in patients with sleep apnea by etanercept, a tumor necrosis factor-alpha antagonist. J Clin Endocrinol Metab 2004;89:4409-13

14 Ciftci TU, Kokturk O, Bukan N, Bilgihan A. The relationship between serum cytokine levels with obesity and obstructive sleep apnea syndrome. Cytokine 2004;28:87-91

15 Querido JS, Sheel AW, Cheema R, Van Eeden S, Mulgrew AT, Ayas NT. Effects of 10 days of modest intermittent hypoxia on circulating measures of inflammation in healthy humans. Sleep Breath 2012;16:657-62

16 Aihara K, Oga T, Chihara Y, Harada Y, Tanizawa K, Handa T et al. Analysis of systemic and airway inflammation in obstructive sleep apnea. Sleep Breath 2013;17:597-604

17 Xie X, Pan L, Ren D, Du C, Guo Y. Effects of continuous positive airway pressure therapy on systemic inflammation in obstructive sleep apnea: a meta-analysis. Sleep Med 2013;14: 1139-50

18 Epstein LJ, Kristo D, Strollo PJ Jr, Friedman N, Malhotra A, Patil SP et al. Clinical guideline for the evaluation, management and long-term care of obstructive sleep apnea in adults. J Clin Sleep Med 2009;5:263-76

19 Gurubhagavatula I, Fields BG, Morales CR, Hurley S, Pien GW, Wick LC et al. Screening for severe obstructive sleep apnea syndrome in hypertensive outpatients. $J$ Clin Hypertens (Greenwich) 2013;15:279-88

20 Senn O, Brack T, Russi EW, Bloch KE. A continuous positive airway pressure trial as a novel approach to the diagnosis of the obstructive sleep apnea syndrome. Chest 2006;129:67-75

Address for correspondence:

Dr Pengju Wang,

Department of Otolaryngology,

Xiangyang Central Hospital Affiliated to Hubei University of

Arts and Science,

Jingzhou Street 39

Xiangyang 441021, PR China

E-mail: xfjh1975@gmail.com

Dr P Wang takes responsibility for the integrity of the content of the paper

Competing interests: None declared 